\title{
THE FEULGEN REACTION APPLIED TO CLINICAL HAEMATOLOGY
}

\author{
BY \\ C. GARDIKAS AND M. C. G. ISRAELS \\ Department of Haematology, University and Royal Infirmary, Manchester
}

(RECEIVED FOK PUBLICATION, MARCH 22, 1948)

The Feulgen reaction is a cytochemical test for the detection of desoxyribonucleic (thymonucleic) acid. After acid hydrolysis, desoxyribonucleic acid in the presence of sulphurous acid gives a characteristic red-purple colour with basic fuchsin, and the depth of the colour roughly reflects the concentration of the compound. The detailed chemistry of the reaction has been fully described by Baker (1942). The reaction has been known for fifteen years and is much used in biology, especially in genetics, but it has only recently been applied to the problems of clinical haematology: La Cour in 1944 used the reaction to study mitotic abnormalities that occur in the bone marrow cells in pernicious anaemia ; Thorell working in Caspersson's laboratory in Stockholm used the Feulgen reaction together with ultra-violet absorption techniques for studying the intimate metabolism of nucleoproteins in blood-forming cells (Thorell, 1944, 1947). We have utilized the Feulgen reaction to give help in classifying blood and bone-marrow cells when the differentiation is of clinical importance and when the routine methods fail to show really clear separation between the different types of cells.

\section{Details of Technical Method}

The technique is a slight modification of those proposed by Rafalko (1946) and Darlington and La Cour (1942).

Air-dried smears of blood or bone-marrow are used and should be fixed as soon as possible, not more than one hour after preparation. The bone-marrow is obtained by sternal biopsy. The fixing solution is composed of: methyl alcohol 15 parts, 5 per cent acetic acid 5 parts, formalin (40 per cent formaldehyde) 1 part, and water 5 parts. The smears are immersed in this fixative for 10 minutes and then treated as follows:

1. Wash in tap water for $\mathbf{1 0}$ or $\mathbf{2 0}$ minutes.

2. Wash in distilled water for 2 minutes.
3. Place in $\mathrm{N} / \mathrm{HCl}$ at room temperature for minutes, then (4) in $\mathrm{N} / \mathrm{HCl}$ at $60^{\circ} \mathrm{C}$. for 8 or $1 \theta$ minutes, and (5) rinse with $\mathrm{N} / \mathrm{HCl}$ at room temperature.

6. Rinse with distilled water.

7. Stain with leucobasic fuchsin solution (vide infra) for $1 \frac{1}{2}$ to 2 hours.

8. Put through two or three changes of $\mathrm{SO}_{2}$ water (vide infra) for 1 or 2 minutes.

9. Wash in tap water for 10 to 15 minutes.

10. Then dehydrate by immersing in 40 per cento 80 per cent, and finally absolute alcohol for 5 minutes each.

11. Clear with xylol.

12. Mount in a neutral synthetic mounting medium

The leucobasic fuchsin is prepared as follows: a 0.5 . per cent solution of basic fuchsin is decolorized by bubbling $\mathrm{SO}_{2}$ through it for 1 hour; if decolorization is not then complete, activated charcoal $(1 \mathrm{~g}$. to $100 \mathrm{ml} \Phi$ should be added to it, and it is shaken and filtered.

The $\mathrm{SO}_{2}$ water is prepared by bubbling $\mathrm{SO}_{2}$ from a syphon through distilled water until a saturate solution is obtained.

It is sometimes helpful to counterstain with Jenner's stain to outline the cytoplasm and stain cytoplasmif granules. This can be done without interfering witk. the Feulgen stain. The Jenner's stain then replacef stage 10 of the technique. Nuclear Appearances of Blood Cells when Stained
by Feulgen's Reaction

With this stain the erythrocytes disappear; the leucocytes and erythroblasts take up stain accordes ing to the concentration of desoxyribonucleic acid present in the nucleus; immature cells have $\overrightarrow{6}$ relatively pale coloration, mature cells a deep tint; the cytoplasm does not stain. The pale stained cells are said to be Feulgen-negative, and the deeply-stained cells Feulgen-positive. Nucleof show up well as clear spaces in the nucleus with $\&$ 

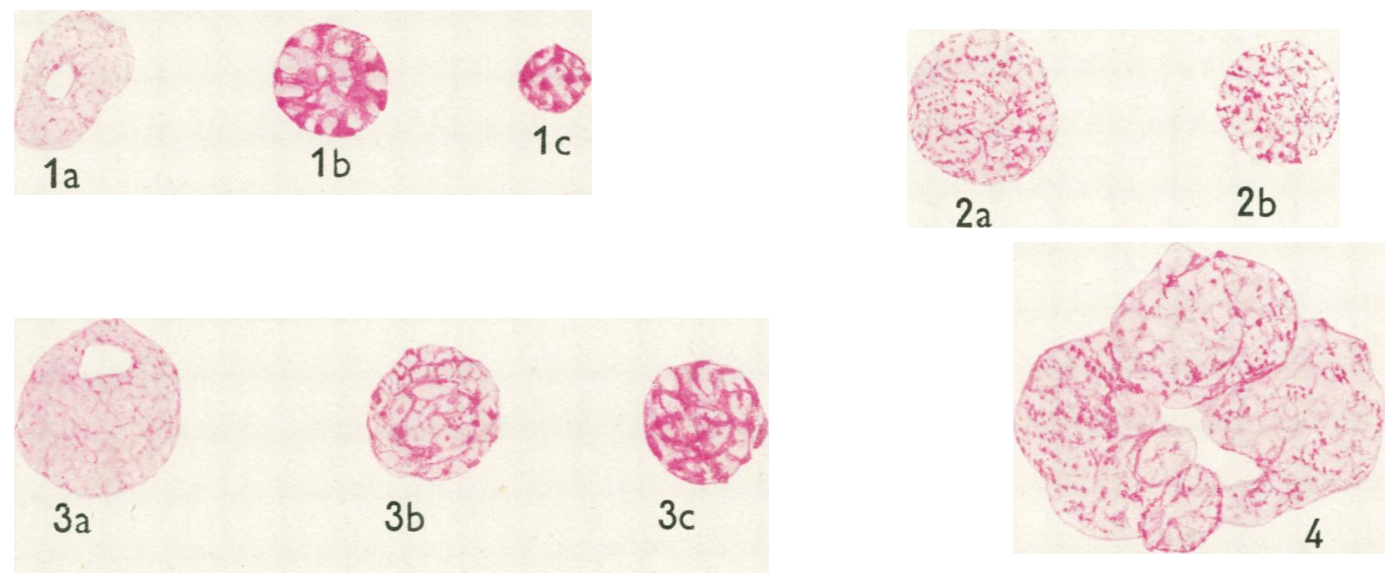

1.-Lymphocytes from a case of chronic lymphatic leukaemia : (a) Lymphoblast, Feulgen-negative, fine chromatin pattern, two nucleoli ; (b) Immature lymphocyte, Feulgen-positive, but shrunken nucleoli still present; (c) Small mature lymphocyte, Feulgen-positive, with chromatin concentrations round the periphery.

2.-Megaloblasts, untreated pernicious anaemia : (a) early megaloblast-note network arrangement of chromatin and absence of nucleoli; (b) intermediate megaloblast, coarser pattern but network still distinct.

3.-Normal erythroblasts : (a) Pro-erythroblast, Feulgen-negative; note large nucleolus and fine but distinct chromatin pattern ; (b) early normoblast, moderate Feulgen reaction ; note coarse concentration of chromatin and absence of nucleoli ; (c) intermediate normoblast, large size, Feulgen-positive with lumpy chromatin pattern.

4.-Megakaryocyte, mature cell moderately Feulgen-positive; no nucleoli.

5.-Granular leucocyte series from chronic myeloid leukaemia : (a) typical myeloblast, Feulgen-negative, four nucleoli ; (b) pro-myelocyte, still Feulgen-negative, only one nucleolus; (c) metamyelocyte, still only moderate Feulgen staining; nucleoli have disappeared; (d) mature polymorphonuclear granulocyte, Feulgen-positive streaks; (e) micromyeloblast : note relatively pale Feulgen stain, fine chromatin pattern and the presence of nucleoli. 
relatively deep staining rim which is more prominent in the more mature cells. The appearances of individual cells are as follows:

Granulocytes.-In the myeloblast, the nucleus takes on a pale stain but the chromatin pattern can often be clearly distinguished; there are two to four large nucleoli clearly detectable as colourless spots. The micromyeloblast is rather more deeply stained but nucleoli are clear. The promyelocyte and myelocyte show changes indicating increasing maturity-the nucleoli decrease in number and size and show a thin surrounding layer of Feulgen-positive material. The metamyelocyte has no nucleoli, but is not so deeply stained as the mature polymorphonuclear, which has Feulgenpositive coarse chromatin with two or three prominent positive spots, usually one in each lobe of the nucleus.

Lymphocytes.-The lymphoblast has a Feulgennegative nucleus of fine structure, not very clearly defined, and there are one or two prominent nucleoli. The immature lymphocyte has a coarser and more deeply staining nuclear chromatin, but nucleoli are still present though small. The nucleus of the mature lymphocyte is composed of Feulgenpositive blocks of chromatin arranged mainly around the periphery.

Monocytes.-The mature monocyte has a fine chromatin structure and stains a faint pink. No aggregations of chromatin occur, and there are no nucleoli.

Erythroblasts (Nomenclature according to Israëls, 1941).-The pro-erythroblast shows a distinct chromatin network but is Fuelgennegative; nucleoli are prominent and are distinguished by a deeply stained surrounding layer.

The normoblasts show early appearance of Feulgen-positive material ; even the early stage is quite deeply stained and shows very well the coarsening and clumping of the chromatin strands. The intermediate normoblasts are fully Feulgenpositive and show the typical coarse chromatin structure.

In the megaloblast series the fine network nuclear structure shows up very strikingly when this technique is used. The early stages show only moderate Feulgen staining ; the nuclear strands of the intermediate stage are fully Feulgen-positive.

Megakaryocytes.-The large nucleus of the mature cell is moderately Feulgen-positive and no nucleoli are present.

\section{Discussion}

The Feulgen reaction has thus several uses as a supplementary technique in clinical haematology.
1. It gives a clear decision about the stage of maturity of a given cell. This is very important in the leukaemias. A not uncommon cause of incorrect diagnosis is confusion between small myeloblasts (micromyeloblasts) and lymphocytes. Sometimes with ordinary stains the differentiation is very difficult even with a good technique ; in particular, nucleoli are often obscured by Romanowsky stains. Schulten (1937), for example, shows on Table 13 in his Atlas a group of cells that he cannot clearly define as myeloblasts or lymphocytes. The Feulgen stain distinguishes clearly between the Feulgen-negative myeloblasts with well-defined nucleoli and the Feulgen-positive lymphocytes without nucleoli.

In chronic myeloid leukaemia the presence of myeloblasts can be used to assess the progress of a case and response to treatment; if these immature cells are few and their proportion decreases with treatment, the prognosis is good. It is much more difficult to assess a case of lymphatic leuleuamia in this way, because with Jenner-Giemsa stain it is not a simple matter to detect immature lymphocytes. The Feulgen stain provides the necessary information, as the proportion of negative or weakly positive lymphoblasts and immature lymphocytes with detectable nucleoli is readily determined and these cells contrast well with the Feulgen-positive mature lymphocytes without nucleoli.

2. The characteristic difference between normoblasts and megaloblast is in the nuclear structure, the chromatin being arranged respectively in clumps or in network of strands. The Feulgen stain gives a sharper picture of the intimate nuclear structure than is possible with Jenner-Giemsa, and another difference is that the normoblasts become more deeply stained at an earlier stage of development than the megaloblasts.

3. The differentiation between lymphoblasts and myeloblasts is a problem as old as clinical haematology, and many techniques-dark-ground illumination, supravital staining, oxidase stainshave been applied with indifferent success. The Feulgen reaction helps because it defines nucleoli. Naegeli (1923) pointed out that, whereas lymphoblasts have few nucleoli, in myeloblasts they are usually multiple. In our experience there are usually one or two nucleoli in lymphoblasts, rarely three; whereas in myeloblasts there are two to four nucleoli, most commonly three. Nucleoluscounting can be a difficult task with JennerGiemsa-stained preparations; with Feulgen stain it is an easy matter to see whether the majority of the immature cells have few or many nucleoli. The presence of a majority of cells with three or 
more nucleoli suggests a myeloblastic form of leukaemia.

The nucleoli with their rim of dark-staining material are a typical feature of the Feulgenstained cells. They appear often much larger than in the Jenner-Giemsa stained preparations. Thorell has related the number and size of the nucleoli to the amount of cytoplasm that is to be formed by the cell in the course of development. The myeloblasts of acute leukaemia often have numerous and prominent nucleoli, yet fail to develop cytoplasm. Two possible explanations are that the cells are abnormal, like malignant cells, and do not develop normally; or that they are normal cells in an abnormal environment. In support of the latter explanation are Israëls' (1940) observation that leukaemic myeloblasts in culture could mature into myeiocytes, and Schwind's (1947) claim that injection of normal blood plasma tends to decrease the proportion of myeloblasts in the blood. It is therefore likely that in pathological states the connexion between nucleolar mass and cytoplasm formation is disturbed.

\section{Summary}

1. Details are given of a rapid technique for applying the Feulgen reaction to blood and bonemarrow cells.

2. The appearances of the more important blood and bone-marrow cells when stained by this Feulgen technique are described and illustrated.
3. The Feulgen reaction is a valuable supplementary technique in clinical haematology for the following reasons:

(a) It distinguishes between immature and mature cells, especially between lymphocytes and micromyeloblasts, and it can be used to assess the proportion of immature cells in lymphatic leukaemia.

(b) The nuclear differences between normoblasts and megaloblasts are sharply defined.

(c) It defines nucleoli very clearly and can therefore aid in distinguishing lymphoblasts with one or two nucleoli from myeloblasts with two to four nucleoli.

4. The suggested correlation between number and size of nucleoli and subsequent cytoplasm formation appears to be disturbed in disease.

The plate was painted by Miss D. Davison, medical artist to the University.

\section{REFERENCES}

Baker, J. R. (1942). In Bourne's Cytology and Cell Physiology, p. 2i. Oxford.

Darlington, C. D., and La Cour, L. F. (1942). Handling of Chromosomes, pp. 110, 122 . London.

Israěls, M. C. G. (1940). J. Path. Bact., 51, 235.

Israels, M. C. G. (1940). J. Path. Bact.,
Israels, M. C. G. (1941). Lancet, 2, 207.

Israels, M. C. G. (1941). $\begin{aligned} & \text { Lancet, } 2,207 . \\ & \text { La Cour, L. F. (1944). Proc. roy. Soc. Edinb., Sect. B., } 62,73 .\end{aligned}$

Naegeli, O. (1923). Blutkrankheiten und Blutdiagnostik. Third ed. Berlin.

Rafalko, J. S. (1946). Stain Technology, 21, 91.

Schulten, H. (1937). Die Sternalpunktion als diagnostiche Methode. Leipzig.

Schwind, J. L. (1947). Amer. J. med. Sci., 213, 170.

Thorell, B. (1944). Acta med. scand., 117, 334 .

Thorell, B. (1947). Acta med. scand., Suppl., 200. 